\author{
Military Technical College \\ Kobry El-Kobbah, \\ Cairo, Egypt.
}

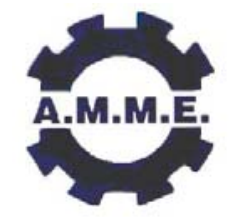

\title{
MACHINING PROCESS PLANNING THROUGH LATENT VARIABLE MODEL INVERSION
}

\author{
HUSSEIN ${ }^{1}$ W.M., MAC-GREGOR ${ }^{2}$ J.F., MANSOUR ${ }^{3}$ D.M.M. and ELBESTAWI ${ }^{4}$ M.A.
}

\begin{abstract}
Manufacturers are always exerting significant effort to improve the quality of machined parts by suitable choice of process parameters. Furthermore, there is a trend within industry to improve process performance and product quality through analyzing available historical data especially in chemical industry. This trend is driven by the need to reduce product development time and cost. The use of latent variable modeling using historical data has been proposed in the past for product design and quality improvement (C.M. Jaeckle and J.F. MacGregor) [23]. This paper outlines the application of such approach using Projection to Latent Structure (PLS) and its model inversion to facilitate the choice of cutting parameters for a desired surface roughness while maximizing the Metal Removal Rate (MRR). The approach is mainly based on using historical data readily available on most of factory platform and simulated through experiments conducted on three different milling machines under normal conditions (sharp tool and stable cut). The model inversion approach is formulated in an optimization problem using the latent space linear model with nonlinear constraint. The approach output solutions were validated with the results showing that the proposed technique can be used for process planning and quality improvement of machining data.
\end{abstract}

\section{KEY WORDS}

Model inversion, Process planning, milling, surface roughness modeling, multivariate analysis.

1 Egyptian Armed Forces.

2, and 4 McMaster University, Hamilton, Canada.

3 Ain shams University, Cairo, Egypt 


\section{INTRODUCTION}

Manufacturers are always exerting significant effort to improve the quality of machined parts using available resources. The machined part quality depends mainly on the process used and the dynamics of the machine as well as the condition of the tool. Experimentally driven process improvement plans typically have high investment costs and are not always successful. A reliable solution to this problem is the application of a model-based approach, which includes the variability in the process parameters that affect the quality of machined parts. Detailed model-based approach is hard to obtain due to the complex nature of the machining process. A fast and a better solution is represented by the use of multivariate latent method. This approach is compatible with the recent trend within industry to improve process performance and product quality through analyzing available historical data. This is especially common in the chemical processing industry. A machined part's quality is determined by different aspects like dimension, surface finish and integrity resulting from the process. Surface finish is commonly specified in industrial applications and it can be described by many parameters like waviness, roughness, lay, directionality and flaws. Surface roughness is generally used to measure surface finish because of its relation to important attributes like friction, fatigue and part appearance. The main parameters that control the cutting action and provide the desired quality level are the rotational speed of the cutter, the feed rate and the depth of cut. Other factors that influence surface finish include the tool/workpiece material and the forces generated, including the dynamic effects, as well as the wear state of the tool. These control parameters have to be selected carefully to set optimum cutting parameters during process planning and to assist in the completion of advanced computer techniques e.g. computer integrated manufacturing systems (CIM).

Many models are currently available in the literature that can predict surface roughness. Abdou et al. [1] proposed a heuristic approach to determine the optimal operating parameters for high speed milling machines. Kopac et al. [2] investigated the use of response surface methodology (RSM) in developing a surface roughness prediction model. Also, Sundaram and Lambert [3] used the RSM for predicting surface roughness in a turning operation. El Baradie [4] and Sahin et al. [5] introduced a surface roughness model for turning: grey cast iron, glass-ceramic and mild steel using carbide tools. Their models were based on statistically designed experiments using regression analysis. Furthermore, Mansour et al. [6] presented a surface roughness model for an end milling operation using the same approach. Similar models based on the design of experiments can be found in [7-10]. Suresh et al.[11] presented the use of genetic algorithms to optimize surface roughness predictions. The dimensional accuracy of aluminum alloy parts was investigated by Fuh and Chang [12] for a peripheral milling operation. Elbestawi et al.[13] investigated the surface topography characteristics of steel workpieces in finish machining using end mills through computer simulation and cutting tests for CAD/CAM implementation. A computer-software was developed by Hashmi et al. [14] to emulate the normal calculations of a turning process using fuzzy logic techniques to predict the optimum cutting parameters. Also, Turkay et al. [15] presented an integrated approach of two groups of optimization processes to facilitate the determination of optimal cutting tools and parameters. A more prevailing technique involves the use of Artificial Neural Networks (ANN) and its combination with other methods. Azouzi and Guillot [16] used an ANN to estimate surface roughness and part dimensions using depth of cut, feed, and radial force with an accuracy of $96 \%$. Also, Tsai et al. [17] used accelerometer and proximity sensor signals to recognize the 
surface roughness produced by different cutting conditions with almost the same accuracy. ANN techniques are characterized as black box approaches which model the relation between different variables to a desired response without giving any information about what happens inside the process. A fundamental approach using a model-based solution is to calculate the surface finish based on using the height of a tooth mark above the point at the lowest level $(h)$ as derived by Martellotti [18] in milling using the following equation [18-19]:

$$
h=\frac{F_{t}^{2}}{8\left(R+F_{t} \times T / \pi\right)}
$$

where $T$ is the number of teeth on the cutter, $R$ is the radius of the cutter; $F_{t}$ is the feed per tooth. The same equation is verified by modeling the exact motion of a milling cutter as reported by Montgomery and Altintas [20]. Moreover, Lee and Dornfeld [21] studied the surface roughness $\mathrm{Ra}$ prediction for a micro-end-milling application using regression analysis. They investigated the effect of run out on the generated surface. Surface roughness parameter $\mathrm{Ra}$ is analytically calculated by the following equation [21]:

$$
R_{a}=\frac{f_{t}^{2}}{32\left(R \pm f_{t} n_{t} / \pi\right)}
$$

where $\mathrm{Ra}$ is the surface roughness, $\mathrm{n}_{\mathrm{t}}$ is the number of teeth on the cutter, $\mathrm{R}$ is the radius of the cutter, $f_{t}$ is the feed per revolution, and the +ive sign refers to up-milling and the -ive sign refers to down milling. But this equation does not consider many factors of machine tool system dynamics and non-linearities. Moreover, there is a lack of robust on-line roughness monitoring. These issues make it very difficult to assess the quality level achieved during the machining process. In addition, during machining, gradual tool wear affects the machined surface quality and contributes to the variation of process parameters like tool life and cutting forces. However, none of these models predict surface roughness at very high cutting speeds while combining all the process parameter variations (e.g. tilt, deflection, run out and wear of the cutting tool) in a single model.

Recently, another approach has been emerging to improve product quality and design through analyzing historical data. Moteki and Arai [22] investigated the use of Principal Component Analysis (PCA) with theoretical modeling to achieve new grades of polyethylene in an LDPE process. Jaeckle and MacGregor [23-24] introduced the use of a latent variable regression (LVR) model inversion technique to obtain the set of operating parameters from historical data, that would give specific level of product quality. Their work was the foundation of null space; a space that gives different operating conditions with the same quality characteristics inside the data boundary. Their technique is illustrated in next section. This foundation was extended in a quadratic optimization form with some constraints by Yacoub and MacGregor [25] and Garcia and MacGregor [26]. They considered different industrial application like linear low density polyethylene (LLDPE), semi-batch reactor, over-molding injection process and pulp digester batch processes.

Attention has so far been directed towards a specific process (e.g. turning or milling) using one machine center, while little effort has been given to milling process and no 
work has been reported on individual units (machine tools) working in cells like unmanned or partially manned production lines. In this work, the latent variable model inversion proposed by Jaeckle and MacGregor [23-24] is applied to an end milling process (side cut) using data from three machine tool centers. Analysis is applied to calculate the null space which aids in selecting machines and different cutting parameters to achieve a desired surface roughness $\mathrm{Ra}$ while maximizing the Metal Removal Rate (MRR). The analysis is extended in an optimization form with a constraint on Hotelling's $\mathrm{T}^{2}$ to get a unique solution and compared with the output from the direct model inversion. This work presents an original application in this field and presents a quick solution for process planning and quality improvement using available historical data. Furthermore, this approaches as mentioned in Jaeckle and MacGregor [23-24], can be used for product transfer between factory plants and is illustrated on different machine tools.

\section{LATENT VARIABLE MODEL INVERSION}

Machined parts can often be produced using a range of cutting parameters that produce a desired surface finish. Also a number of different machines operating under different conditions can be used to get the desired final product quality. In this case each machine has a combination of operating conditions such as speed, feed, depth of cut, and tool condition for example that result in a part with the desired quality. This choice is driven by the need to minimize cost while achieving high productivity. Currently available theoretical models for surface roughness do not take into account the dynamics of the process. However this information is contained within the historical data base for each machine.

Empirical solutions using standard regression values reported in the literature model the quality (surface roughness Ra) space (Y's) only and do not take into account the correlation structure between the input $X$ variables such as the cutting parameters. Multivariate latent variable modeling techniques like Partial Least Squares or Projection to Latent Structure (PLS) provide models for both the input X-space and the output Yspace by extracting the main underlying orthogonal directions of variation in the correlated data and then using this information to find the maximum correlation between the two spaces. These models can then be defined as generalized regression methods that work on reduced rank matrices. They are generally applied for data analysis which takes maximum benefit from the historical data available in most industrial settings and tries to extract useful information from it.

\section{Methodology}

First summarize the problem by setting $Y=\beta X+\varepsilon$, the standard regression form, where $Y$ can be surface roughness of the machined parts and $X$ can be feed/tooth, rpm, depth of cut, measured forces, vibration, etc. Here, the analysis is to get $X^{\prime}$ 's when $Y$ is already known. The normal least square solution is $x_{\text {new }}^{T}=y_{\text {des }}^{T} \cdot\left(\hat{\beta}^{T} \cdot \hat{\beta}\right)^{-1} \cdot \hat{\beta}^{T}$ which has many shortcomings like $X$-matrix ill conditioning and cases where there is no correlation structure between the $\mathrm{X}$-variables and $\mathrm{Y}$-variables (in case we have more than quality variables describing an output) which cannot assure the reliability of the new conditions with the past process conditions [23]. This serious problem arises because this method is a data driven technique which must constrain the solution to the past historical data 
region where the model was built. Jaeckle and MacGregor's [23-24] procedure is illustrated in the following steps:

1- Mean centering and scaling data matrices $X$ and $Y$.

2- Building the latent model : $\hat{X}=T \cdot P^{T}$ and $\hat{Y}=T \cdot B$

3- Decomposing $X$ using singular value decomposition (SVD): $X=U_{A} \cdot \sum_{A} \cdot P_{A}^{T}$, where $A$ is the number of effective principal components, $U_{A}$ has orthonormal columns and $\Sigma_{A}$ is a diagonal matrix with a-th largest singular value of $X$. By analogy with step 2 for $X$-equation we can express the score " $T$ "as : $T=U_{A} \cdot \Sigma_{A}$

4- In case we deal with more than $Y$-variables (e.g. surface roughness and part dimensions), we must ensure that the new desired $Y_{\text {des }}$ has the same correlation structure as the available $Y$-data. This means keeping the new desired output with the process capability. This aspect can be tested by performing principal component analysis on the $Y$-matrix including the new desired $Y_{\text {des. }}$. Also, it means that the new $Y_{\text {des }}$ should have low DModX (residuals).

5- The model inversion is then applied using the pseudo-inverse to " $B$ " by the following manner under three conditions using equations in steps $2 \& 3$ to get :

$$
y_{\text {des }}^{T}=u_{\text {new }}^{T} \cdot \sum_{A} \cdot B \Rightarrow \hat{u}_{\text {new }}^{T}=y_{\text {des }}^{T} \cdot B^{T} \cdot\left(B \cdot B^{T}\right)^{-1} \cdot \sum_{A}^{-1} \Rightarrow \hat{x}_{\text {new }}^{T}=\hat{u}_{\text {new }}^{T} \cdot \sum_{A} \cdot P_{A}^{T}
$$

This way of calculating the new score for the desired $Y_{\text {des }}$ then estimating the operating conditions $X_{\text {new }}$, to assure the retention of the correlation structure based on the past experienced data.

6- Jaeckle and MacGregor [23-24] reported three scenarios to solve this design problem, where $\mathrm{k}$ is the $\mathrm{Y}$ 's dimension and $\mathrm{A}$ the number of effective principal components:

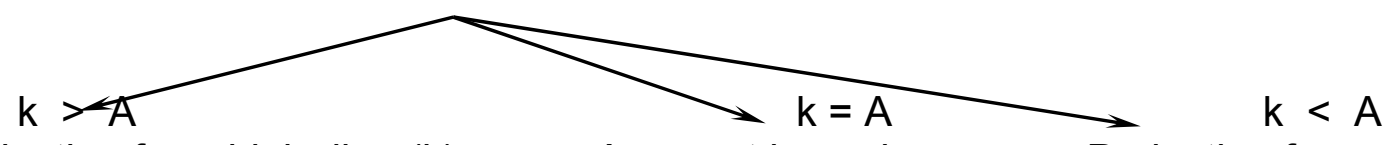

Projection from high dim. $(\mathrm{k})$ to lower $\operatorname{dim}$. (A) and this will be simple inversion.

An exact inversion

Projection from

lower dim. (k) to

higher $\operatorname{dim}$. (A)

The last situation is our case and the most common to happen. It infers that the extra $X$ - dimensions (which have no influence on $Y$ ) define a space of solutions which yield the same output response $Y$ and we call it the "null space".

7- Null space construction is based on adding a new term $x_{\text {null }}$ to the existing estimated $x_{n e w}$ without affecting or changing the $y_{\text {des. }}$. This is obtained by augmenting the estimated new process conditions with the null term:

$$
x_{\text {pred }}^{T}=\hat{x}_{\text {new }}^{T}+x_{\text {null }}^{T} \Rightarrow x_{\text {pred }}^{T}=\left(\hat{u}_{\text {new }}^{T}+u_{\text {null }}^{T}\right) \cdot \sum_{A} \cdot P_{A}^{T}
$$

- To not change the value of $y_{\text {des }}^{T}$ and we have $\hat{u}_{\text {new }}^{T} \cdot \Sigma_{A} \cdot B=y_{\text {des }}^{T}$, this requires that $u_{\text {null }}^{T} \cdot \sum_{A} \cdot B=0$.

- We have $y_{\text {des }}^{T}=u_{\text {new }}^{T} \cdot \sum_{A} \cdot B$, then $B=\sum_{A}^{-1} \cdot U_{A}^{T} \cdot Y$

- Substituting in the null equation : $u_{\text {null }}^{T} \cdot \sum_{A} \cdot \sum_{A}^{-1} \cdot U_{A}^{T} \cdot Y=0$

- We get $u_{\text {null }}^{T} \cdot U_{A}^{T} \cdot Y=0$, so any vector $u_{\text {null }}^{T}$ lies in the left null-space of $U_{A}^{T} \cdot Y$ is a solution to this equation. 
- By singular value decomposition (SVD) of $U_{A}^{T} \cdot Y$ they estimated this space as: $U_{A}^{T} \cdot Y=\left[G_{1} \vdots G_{2}\right] \cdot \sum_{U_{A}^{T} Y} \cdot P_{U_{A}^{T} Y}^{T}$, where $G_{2}^{T}$ defines this space.

- $\quad$ Finally $u_{\text {null }}$ is calculated from : $u_{\text {null }}^{T}=\lambda^{T} \cdot G_{2}^{T}$ where $\lambda^{T}$ is arbitrary in magnitude and direction, and solutions are calculated for different values of $\lambda^{T}$-vectors and only those within the Hotelling's confidence limits are retained.

\section{Process Planning Through PLS Model Inversion of a Side End-Milling Process}

In this section, the model built in [27] will be used for model inversion and optimization applications and will be summarized for illustration purposes. It is the objective of this research to examine an existing approach (multivariate analysis) to facilitate machining process planning (the choice of cutting parameters) and to improve the quality of machined parts (surface roughness) through using available data (historical data) while maximizing the MRR. In this application and because it is a newly proposed system for surface roughness monitoring, a set of experiments were carried out representing past historical data.

This approach is a data driven model (empirical), so it will find a set of parameters that has the same correlation structure and range [23-24-25] inside a bounded region (confidence interval of latent space). Available data (experimental or historical) are always very rich and contains a wide variety of information (e.g. sharp, wear, chatter, process deviation, etc.), except that in this application we intend to improve the process performance and product quality. So, in this work we used only normal data (sharp tool and stable cuts) to build the model. Table 1 illustrates the configuration of the $X-Y$ matrices used in this chapter.

Table1. X-Y matrix

\begin{tabular}{|l|c|c|c|c|c|c|c|c|c|c|c|c|c|}
\hline \multicolumn{10}{|c|}{ Machines } & X-Matrix & Y- \\
\hline & $\mathrm{X} 1$ & $\mathrm{X} 2$ & $\mathrm{X} 3$ & $\mathrm{fx}$ & fy & fz & Vib & AE & Cu & F/t & DOC & rpm & Y=Ra \\
\hline & $\mathrm{M} 1$ & $\mathrm{M} 2$ & $\mathrm{M} 3$ & & & & & & & & & & \\
\hline M1 & 1 & 0 & 0 & & & & & & & & & & \\
\hline M2 & 0 & 1 & 0 & & & & & & & & & & \\
\hline M3 & 0 & 0 & 1 & & & & & & & & & & \\
\hline
\end{tabular}

Again, the multivariate PLS model is a linear model given in matrix form by: $\mathrm{Y}=\mathrm{X} \cdot \mathrm{B}+\varepsilon$. To build the model using process variables, let $\mathrm{X}$ include variables containing the information in the sensory data (forces, vibration, $A E$ and spindle current), and the cutting data (feed/tooth, cutting speed and depth of cut). The main control parameters are illustrated in figure1. These sensors were believed to have a direct correlation with the machined surface roughness and in the same time cover many sources of variations that could happen during the machining process. Then let $Y$ consist of the process output response which is the surface roughness Ra values of the machined parts in this case. 


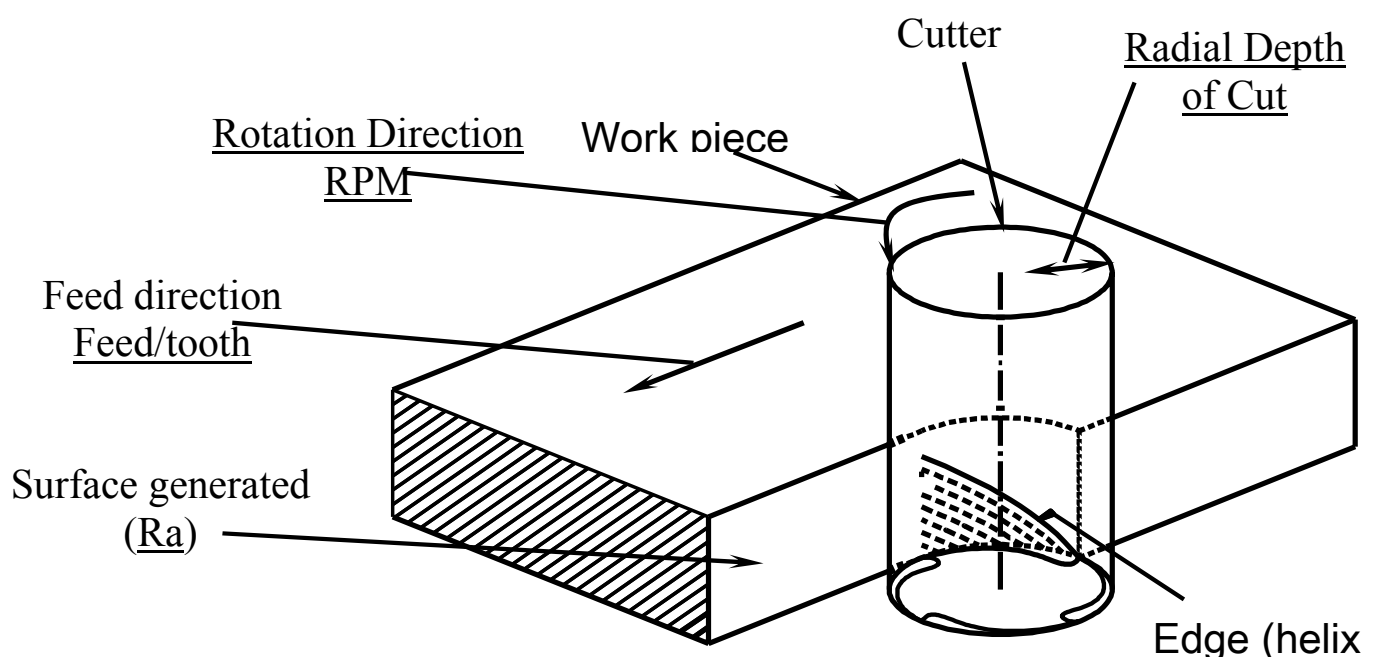

Figure1 Main cutting parameters of end-milling (side-cut) process

Finally, the PLS regression is made between $X$ and $Y$ based on an algorithm which is superficially "similar" to NIPALS. The model and all calculations were carried out using Matlab-7 code. The manipulated parameters are the feed, speed and depth of cut, and the sensor outputs represent the dynamics of each machine which will influence their distribution inside the region of confidence interval. The experiments were based on a set of runs with two combinations of feed/tooth and depth of cut and different steps of cutting speeds with maximum of $15000 \mathrm{rpm}$ (the maximum of the Makino machine tool which is the lowest machine tool rpm). The combinations of feed/tooth and depth of cut were chosen based on the manufacturer's recommendations that give acceptable surface roughness for this application. Although the analysis can be made with different speeds for each machine it will decrease the predictability of the model. Also, it is preferred to make the comparison between different machines operating under the same conditions. The number of runs for each machine was different depending on the maximum capacity of the machine. For example, when running the experiments for the LX1 machine with a maximum rpm $=60,000$, we took a step every $2000 \mathrm{rpm}$ for each combination of feed and depth of cut (with a total of 20 runs to $15000 \mathrm{rpm}$ ). For the Makino machine we took a step every $1000 \mathrm{rpm}$ for the same combinations (with a total of 36 runs). Table 2 illustrates the model-matrix used.

\section{Application: PLS model inversion (one machine tool data)}

The following sections illustrate the concept using data from one machine tool of an end milling process then extending this to involve data from three milling machine tools. As mentioned above we should start by choosing the desired quality aspects and check their feasibility with available past data. In this application we have only one quality aspect, the surface roughness $\mathrm{Ra}$, so we choose an acceptable level which represents an improvement over the existing results which is $\mathrm{Ra}=0.18 \mu \mathrm{m}$. The model was built based on PLS to relate surface roughness $\mathrm{Ra}$ with sensor outputs and the main cutting parameters (feed/tooth, rpm, D.O.C.) using two principal components with the following percentage explanation: $R^{2} X$ (cum): $0.527, R^{2} Y$ (cum): $0.708, Q^{2}$ (cum): 0.555 . The model was built using only normal data (sharp tool) from the Makino machine tool using 34 observations after removing 2 outliers. 
Table 2. Test matrix - Sharp tool

\begin{tabular}{|c|c|c|c|c|}
\hline \multirow[t]{3}{*}{$\begin{array}{l}\text { Cutting speed } \\
\text { (rpm) }\end{array}$} & \multicolumn{2}{|c|}{$\begin{array}{l}\text { Radial depth of cut } \\
0.45(\mathrm{~mm})\end{array}$} & \multicolumn{2}{|c|}{$\begin{array}{l}\text { Radial depth of cut } \\
0.65(\mathrm{~mm})\end{array}$} \\
\hline & \multicolumn{2}{|c|}{ Feed per tooth (mm) } & \multicolumn{2}{|c|}{ Feed per tooth $(\mathrm{mm})$} \\
\hline & 0.05 & 0.075 & 0.05 & 0.075 \\
\hline \multirow{3}{*}{$\begin{array}{c}7,000 \\
\ldots \ldots \\
15,000\end{array}$} & $\bullet$ & - & • & - \\
\hline & $\bullet$ & $\bullet$ & $\bullet$ & $\bullet$ \\
\hline & $\bullet$ & $\bullet$ & $\bullet$ & $\bullet$ \\
\hline \multicolumn{5}{|c|}{ LX1(rpm): 7-9-11-13-15 (20 tests) } \\
\hline \multicolumn{5}{|c|}{ FX5(rpm): 7-9-11-13-15 (20 tests) } \\
\hline \multicolumn{5}{|c|}{ Makino(rpm): 7-8-9-10-11-12-13-14-15 (36 tests) $\ldots$ Where: $r p m \times 1000$} \\
\hline
\end{tabular}

As mentioned above the number of $Y(k=1)$ is less than the number of latent directions in $X$-variables $(A=2)$. In this case we must calculate the remaining direction using the Null space approach. A set of ten $x_{\text {null }}^{T}$ vectors were added to the model inversion solution $\hat{x}_{\text {new }}^{T}$ for the purpose of illustration with only a subset selected if their Hotelling's T2 was within the $99 \%$ confidence interval. In this way the model inversion solution assures that the residuals (DModX) are equal to zero. As shown in figure 2, the null space constitutes a straight line with different vectors (cutting parameters) for the same required surface roughness $\mathrm{Ra}=0.18 \mu \mathrm{m}$. Since it is a linear model it was expected to lie in this position which gives lower Ra values. A set of three runs under the new cutting parameters was carried out and then projected back in the latent space as shown in the same figure. It ended up with an average $\mathrm{Ra}=0.187 \mu$ m which represents an improvement over the existing results of $\mathrm{Ra}=0.192 \mu \mathrm{m}$. This result was expected since the solution obtained shows a significant decrease in feed/tooth which has a great impact on the surface roughness obtained. Moreover, the new cutting parameters show close values especially for the main cutting parameters; there are no significant differences between them as illustrated in Table 3.

In some applications a table dynamometer is not easy to implement in industry, thus the force data was removed. A new model was then fitted which relates surface roughness $\mathrm{Ra}$ with sensor outputs (current, acoustic and vibration) and the main cutting parameters (feed/tooth, rpm, D.O.C.) using two principal components with the following percentage explanation: $R^{2} X$ (cum): 0.677, $R^{2} Y$ (cum): $0.741, Q^{2}$ (cum): 0.697. The same analysis was carried out with null space at $\mathrm{Ra}=0.18 \mu \mathrm{m}$, except here the model results in a wider range of cutting parameters as shown in table 4 . One run of the experiments is shown in figure 3 using new cutting parameters (null vector-8) which end with $\mathrm{Ra}=0.2 \mu \mathrm{m}$ which comes close to the desired one.

This application shows the capability of the approach to improve quality using available data only. Furthermore, it can be used for facilitating the choice of the main cutting parameters for the different desired surface roughness parameters. The idea is that the null space (line) will move to the right depending on the desired level as will be shown in the next section. 
Table 3. Model Inversion output

\begin{tabular}{|c|c|c|c|}
\hline \multicolumn{4}{|c|}{ Model inversion output for $\mathrm{Ra}=0.18 \mu \mathrm{m}$} \\
\hline & $\begin{array}{c}\text { Feed/tooth } \\
(\mathrm{mm})\end{array}$ & Depth Of Cut $(\mathrm{mm})$ & $\mathrm{rpm}$ \\
\hline V4 & 0.0407 & 0.6992 & 7,449 \\
\hline V8 & 0.0396 & 0.4139 & 6,831 \\
\hline M.I. & 0.0402 & 0.5566 & 7,140 \\
\hline \multicolumn{2}{|c|}{ Where: V4 ...Null vector 4, V8 ...Null vector 8, M.I. model inversion solution } \\
\hline
\end{tabular}

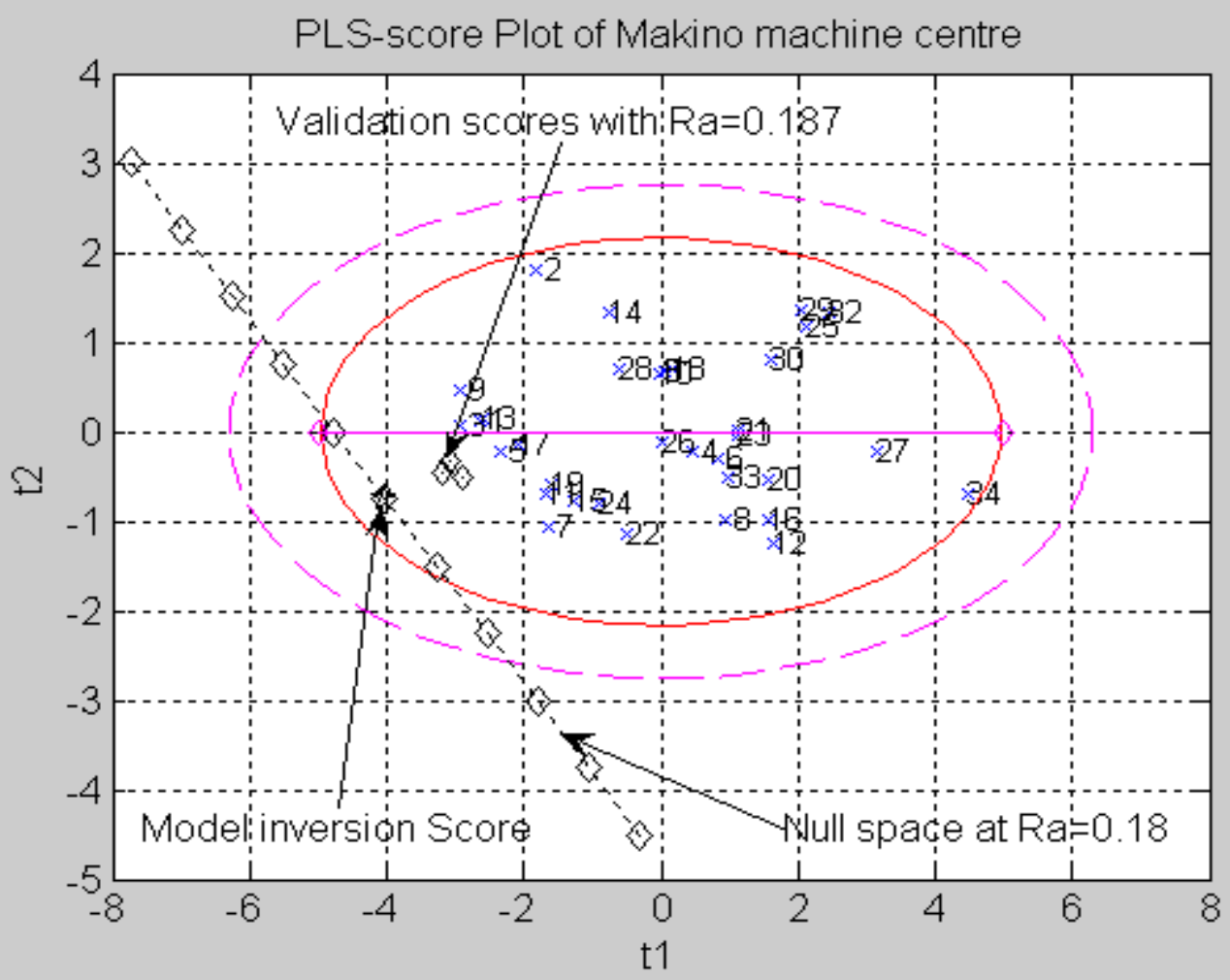

Figure 2. Score plot of Machino PLS-model with null space at $\mathrm{Ra}=0.18 \mu \mathrm{m}$

Table 4. Model Inversion output

\begin{tabular}{|c|c|c|c|}
\hline \multicolumn{4}{|c|}{ Modified PLS-model with model inversion output for $\mathrm{Ra}=0.18 \mu \mathrm{m}$} \\
\hline & Feed/tooth $(\mathrm{mm})$ & Depth Of Cut $(\mathrm{mm})$ & $\mathrm{rpm}$ \\
\hline V4 & 0.0277 & 0.4825 & 11,831 \\
\hline V8 & 0.0507 & 0.5385 & 3,697 \\
\hline M.I. & 0.0392 & 0.5105 & 7,764 \\
\hline
\end{tabular}




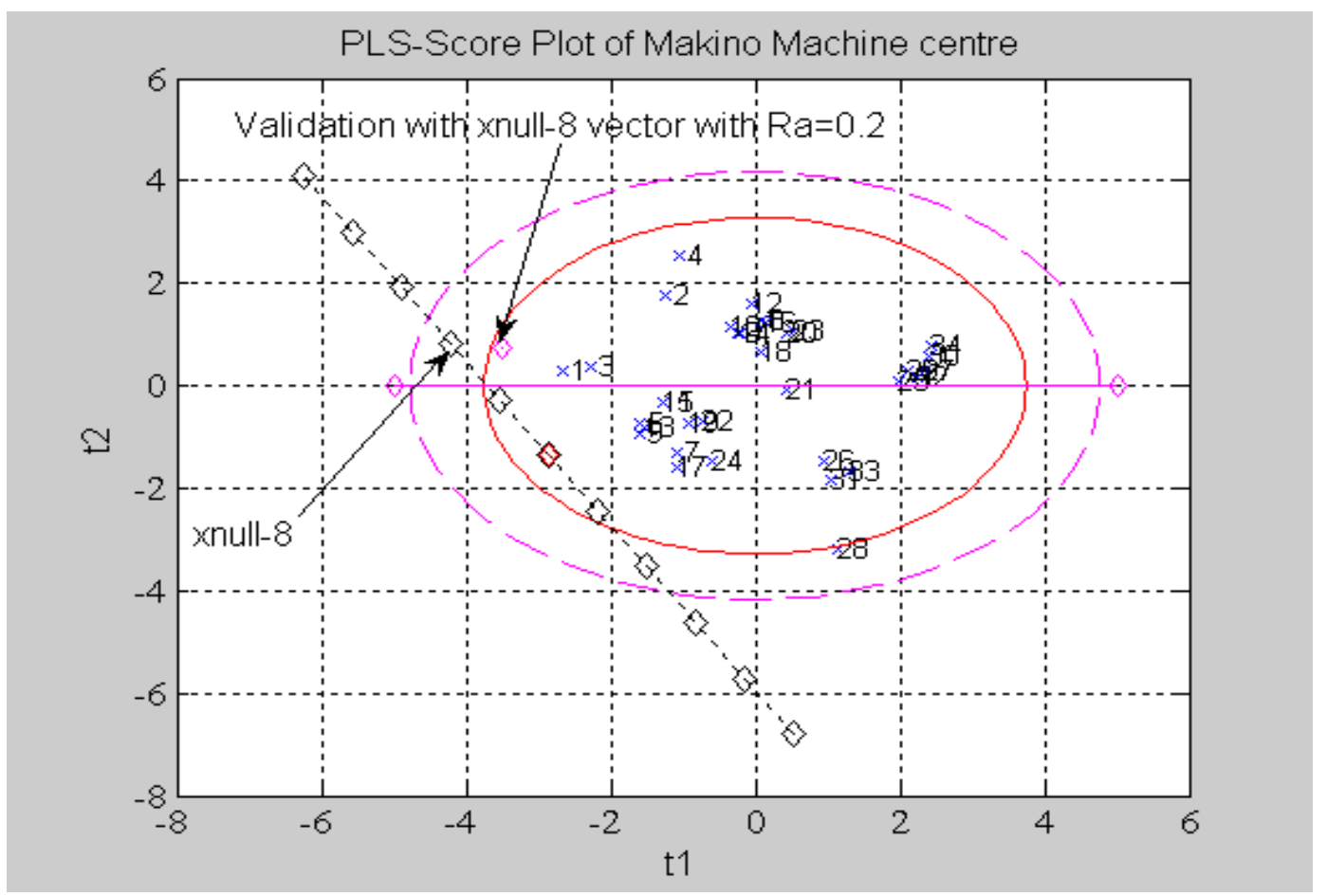

Figure 3. Score plot of Makino modified model with null space at $\mathrm{Ra}=0.18 \mu \mathrm{m}$

\section{Application: PLS model inversion (three machine tools data)}

In this section the same approach is applied on data from three machine tools and the problem is reformulated in an optimization format as a first step to be used in future work to introduce more constraints like machining costs and production rate. A model was fitted based on PLS relating surface roughness $\mathrm{Ra}$ with sensor outputs and the main cutting parameters (feed/tooth, rpm, D.O.C.). In this model we kept dummy variables in the X-matrix to group observations from each machine tool. The model was built using three principal components with the following percentage explanation: $R^{2} X$ (cum): $0.753, R^{2} Y$ (cum): $0.66, Q^{2}$ (cum): 0.586 . The model was built using only normal data (sharp tool) from the three machines that gave acceptable quality as indicated by the measured $R_{a}$ value. Score and loading plots are shown in figures 4-5.

Again a set of ten $x_{\text {null }}^{T}$ vectors were added to the model inversion solution $\hat{x}_{\text {new }}^{T}$ with only a subset selected if their Hotelling's T2 was within the 99\% confidence interval for $\mathrm{Ra}=0.3 \mu \mathrm{m}$. As shown in figure 6 , the null space constitutes a straight line with different vectors (cutting parameters) for the same required surface roughness $\mathrm{Ra}=0.3 \mu \mathrm{m}$. Since it is a linear model it was expected to lie in this position which gives the mean of three machine tool $\mathrm{Ra}$ values. This analysis facilitates process planning using available data by twofold. First, by checking the observations near the null space for each machine (group of observations); it can provide quick information about process conditions to get the desired surface roughness (in this case $0.3 \mu \mathrm{m}$ ) using different machine tools. Table 5 shows some observations from each machine near the null space line.

Secondly, by using the model inversion solution for new conditions (without calculating the null space), it can facilitate the choice of a suitable machine tool to achieve the 


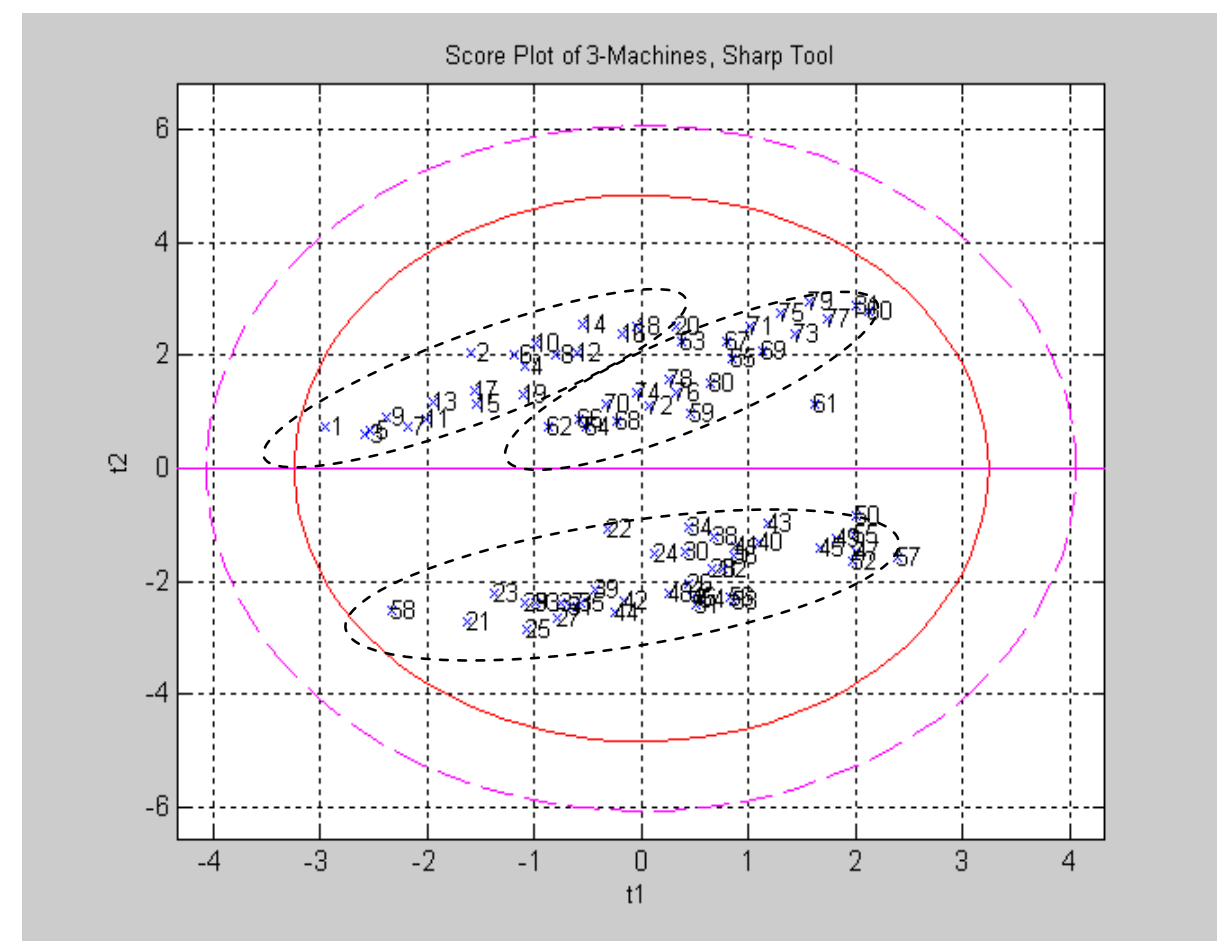

Figure 4. Score plot of data from three machines.

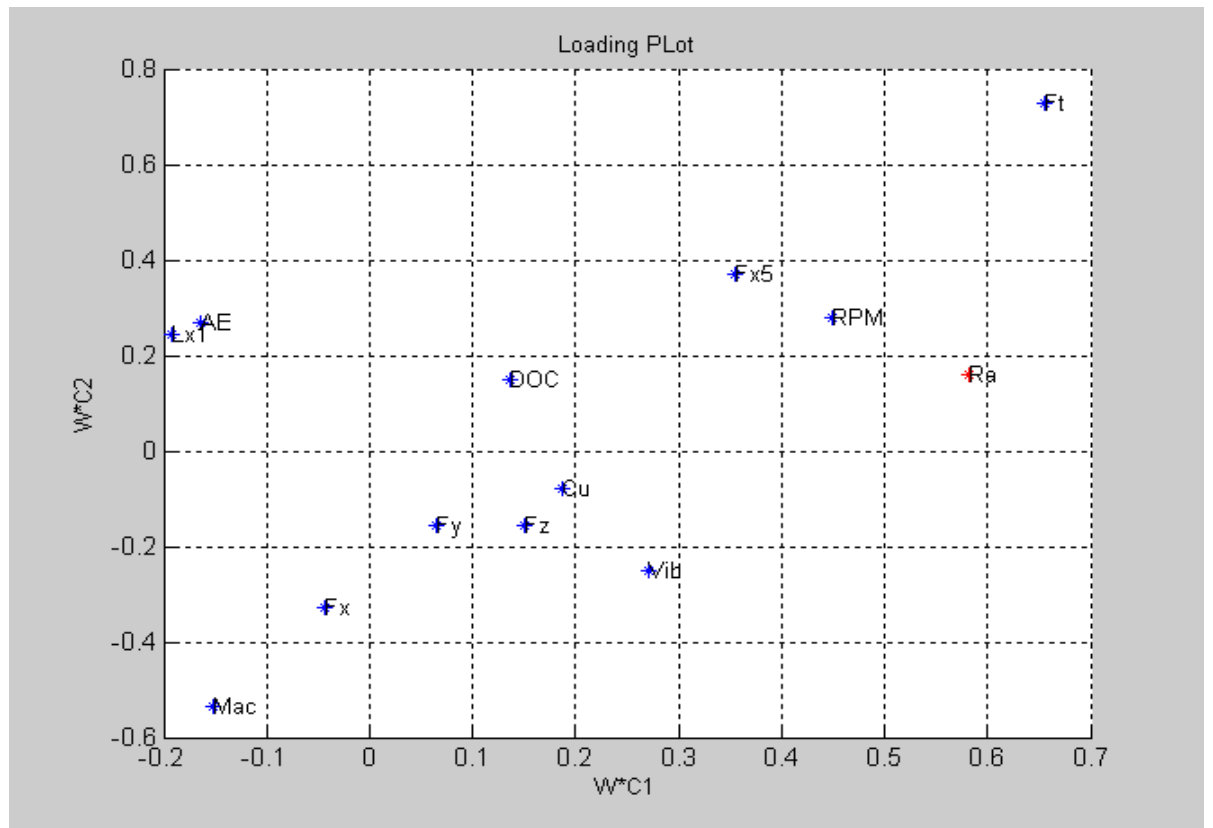

Figure 5. Loading plot of data from three machines.

desired surface roughness. This can be handled as a pattern recognition problem. Out of the many techniques available in pattern recognition, the simple template matching one was chosen. This algorithm involves a distance computation between reference patterns (Machine observations mean) and the input (new estimated condition). Hence, the decision is taken based on the nearest neighbor rule (NN). First, we estimate the mean of each machine tool observation which represents the reference patterns. 
Secondly, recognition of the nearest machine to the new re-designed cutting parameters is carried out by calculation of the Euclidean distance between them and the model inversion solution is shown in figure 6. The decision is taken based on the cumulative Euclidean distances for each machine mean, and the best match is the smallest. Mathematically, it can be expressed by having $P_{m}(n)$ patterns, where $m$ is the number of stored patterns and $n$ the number of frames or points in each stored pattern (machine). In this case we have one point from each machine (the observations mean in the latent space) and three patterns exist from three machine tools. Then the decision rule to get the best match is a minimization approach on the distance between the re-designed parameters and individual machine mean. The Euclidean distance is simply the square root of the sum of the differences squared between each input and the similar stored patterns. The definition of the Euclidean distance is a straight line distance between two points. In a plane with $\mathrm{p}_{1}$ at $\left(\mathrm{x}_{1}, \mathrm{y}_{1}\right)$ and $\mathrm{p}_{2}$ at $\left(\mathrm{x}_{2}, \mathrm{y}_{2}\right)$, it is $\mathrm{Eu}=\sqrt{\left((\mathrm{x} 1-\mathrm{x} 2)^{2}+(\mathrm{y} 1-\mathrm{y} 2)^{2}\right)}$. In this case, the FX5 machine has the minimum distance over the other machine with a nearly equal distance with the Makino machine. Other constraints can be added depending on the process requirements like power consumption and level of vibration, etc.

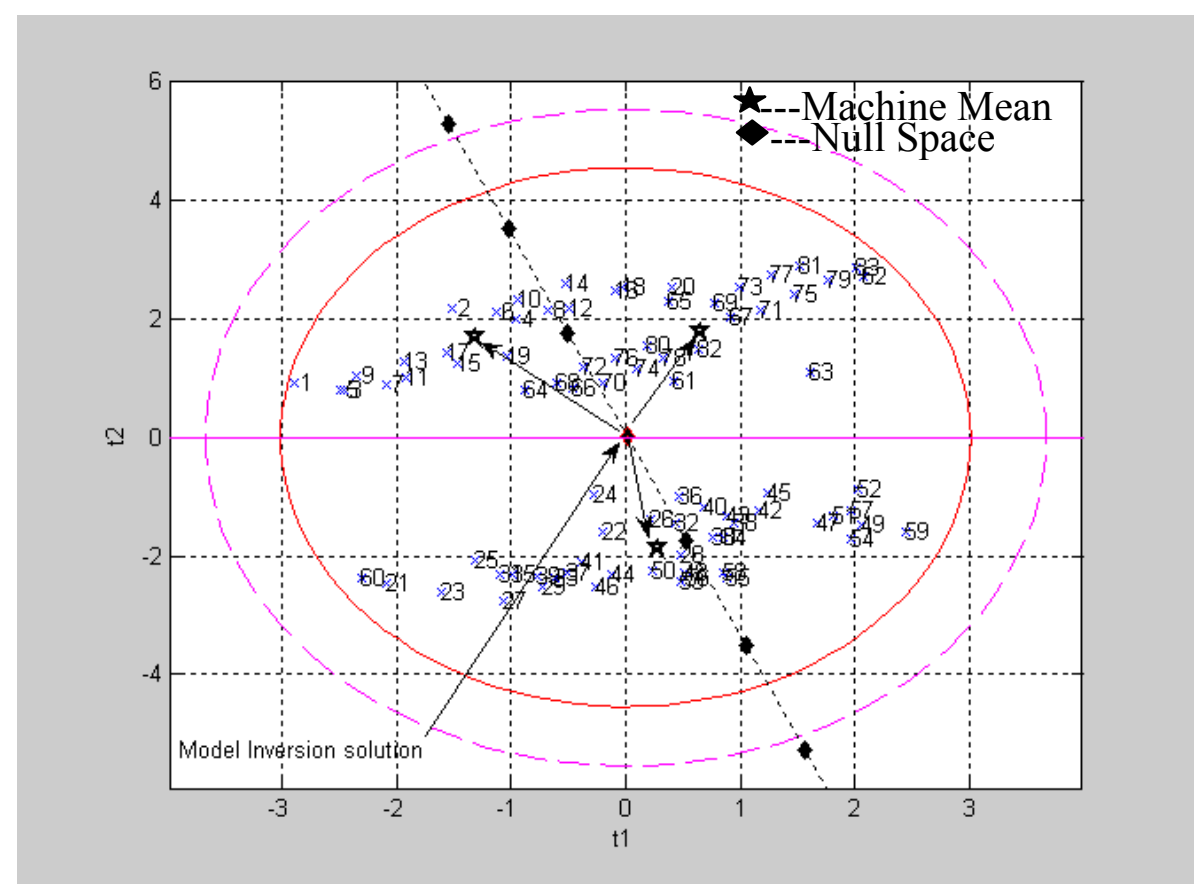

Figure 6. Score plot of data from three machines with Null space at $\mathrm{Ra}=0.3 \mu \mathrm{m}$

Table 5. Observations from each machine with associated Ra

$\begin{array}{lcccc}\text { Obs. } & \text { Feed/tooth }(\mathrm{mm}) & \text { Depth Of Cut }(\mathrm{mm}) & \text { rpm } & \text { Ra }(\mu \mathrm{m}) \\ \text { LX1-8 } & 0.075 & 0.65 & 9,000 & 0.307 \\ \text { LX1-12 } & 0.075 & 0.65 & 10,000 & 0.316 \\ \text { FX5-70 } & 0.05 & 0.65 & 9,000 & 0.292 \\ \text { FX5-72 } & 0.05 & 0.45 & 11,000 & 0.284 \\ \text { Mac-28 } & 0.075 & 0.45 & 8,000 & 0.297 \\ \text { Mac-32 } & 0.075 & 0.45 & 9000 & 0.29\end{array}$




\section{Application: Optimization of PLS model (three machine tools data)}

Optimization in the latent space is another way to formulate the problem like in Yacoub and MacGregor [25] and Garcia and MacGregor [26]. This formulation is just a first step for future work with more constraints on cost of machining, path time and power consumption. Since the latent analysis only builds a correlation structure between parameters at the time when the data was collected, so any optimization solution must be constrained inside this structure and space. This means that the new solutions must be within the range of past data. In this work the problem is formulated using the linear model between the surface roughness $\mathrm{Ra}$ and the manipulated parameters (feed/tooth, depth of cut and rpm) in addition to the sensor outputs (forces, vibration, acoustic emission, current and dummy variables). The approach starts first by setting the objective function to be minimized as:

$$
\begin{aligned}
& \min _{u_{\text {new }}} f(x)=\left(Y_{\text {des }}-Y_{\text {new }}\right)^{T} \cdot\left(Y_{\text {des }}-Y_{\text {new }}\right) \\
& \text { Where } \\
& Y_{\text {des }}=\left[\frac{\left(Y_{\text {des }}-\text { mean }(Y)\right)}{\operatorname{std}(Y)}\right] \\
& Y_{\text {new }}=\left(T \cdot B^{T}\right) \\
& T=u_{\text {new }}{ }^{T} \cdot \sum_{A}
\end{aligned}
$$

Subject to non-linear constraints:

$$
\text { 1- } \quad T^{2}=\sum_{a=1}^{A} \frac{\left(u_{\text {new }}^{T} \cdot \sum_{a}\right)_{\text {new }, a}^{2}}{S_{a}^{2}} \leq \text { const. }
$$

where: $S_{a}^{2}$ is the variance of each corresponding latent score $\tau_{\text {new,a }}$.

Constant is the $95 \%$ or $99 \%$ Hotelling's confidence interval.

$$
\text { 2- } \operatorname{DMod} X=\left[\frac{1}{L-A} \sum_{l=1}^{L}\left(x_{\text {desired }, 1}-\hat{x}_{\text {desired }, l}\right)^{2}\right]^{\frac{1}{2}} \leq \varepsilon
$$

where: $D M o d X$ is the model residual.

and $\varepsilon$ a very small value.

This last constraint can be excluded because it is assumed that the solution will follow the correlation structure in the available data so the residual error DModX will be zero. The second step is to reconstruct the operating conditions $\hat{x}_{\text {new }}$ from $\tau_{\text {new }}$ by the following equation:

$$
\hat{x}_{\text {new }}=u_{\text {new }}^{T} \cdot \sum_{a} \cdot P^{T}
$$

The same model for the three machine data was used and the same analysis was carried in the null space at $\mathrm{Ra}=0.2 \mu \mathrm{m}$, except here the optimization results were added as shown in figure 7 . One run of the experiments is shown using the new cutting parameters (optimization solution) which end with $\mathrm{Ra}=0.21 \mu \mathrm{m}$ which comes close to the desired one. Again this application shows that the optimization solution can be applied to improve quality using historical data only. 


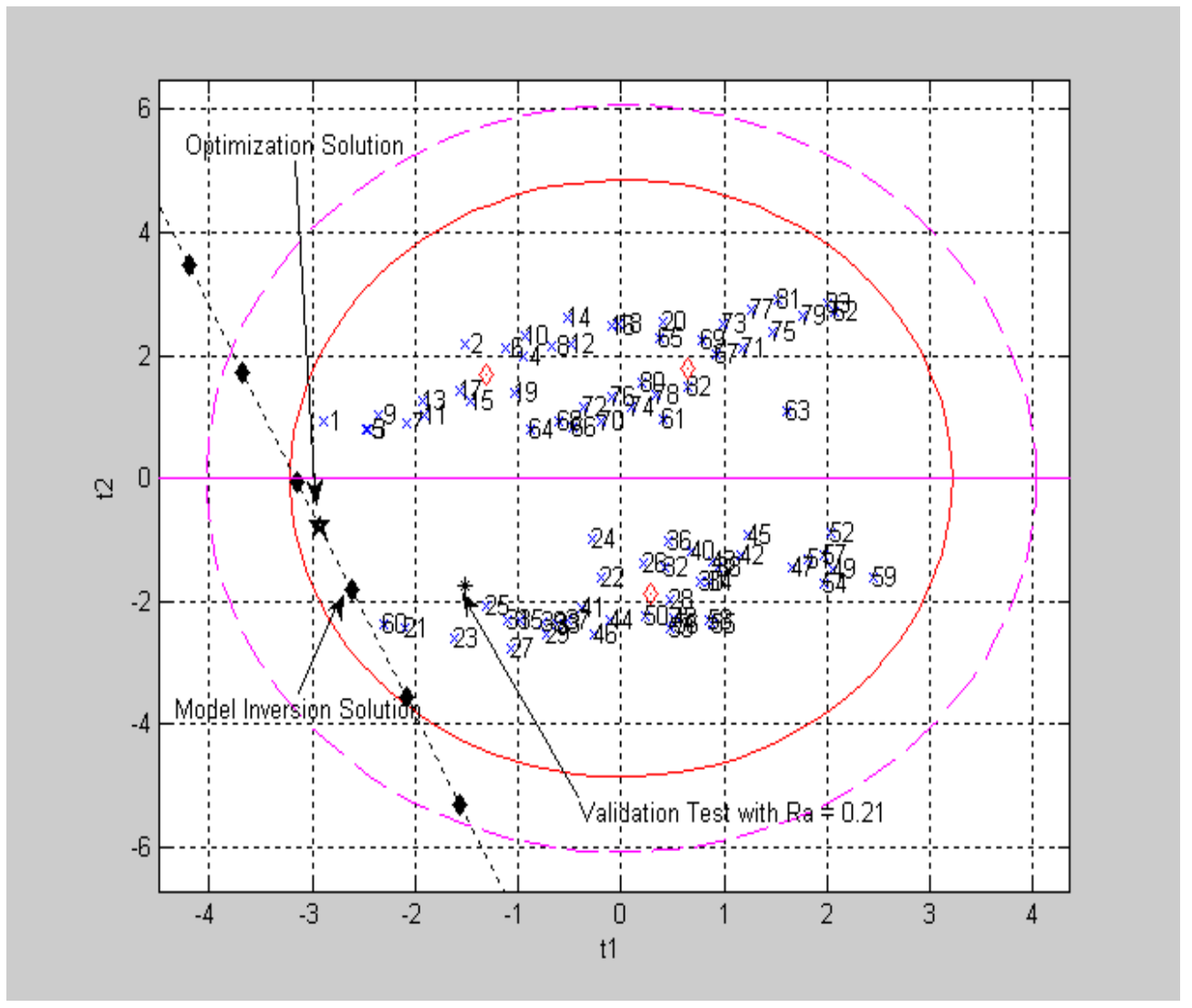

Figure 7 Score plot of data from three machines with optimization, model inversion solutions and validation test for $\mathrm{Ra}=0.2 \mu \mathrm{m}$

A final model was fitted based on PLS results relating surface roughness Ra with sensor outputs and the main cutting parameters (feed/tooth, rpm, D.O.C.). In this model we kept dummy variables in the X-matrix to group observations from each machine tool. In addition, in this application we used different speeds depending on the capability of each machine as shown in Table 6.

Table 6. Test matrix - Sharp tool

\begin{tabular}{|c|c|c|c|c|}
\hline \multirow[t]{3}{*}{$\begin{array}{l}\text { Cutting speed } \\
\qquad(\mathrm{rpm})\end{array}$} & \multirow{2}{*}{\multicolumn{2}{|c|}{$\begin{array}{l}\text { Radial depth of cut } \\
0.45(\mathrm{~mm}) \\
\text { Feed per tooth }(\mathrm{mm})\end{array}$}} & \multirow{2}{*}{\multicolumn{2}{|c|}{$\begin{array}{c}\text { Radial depth of cut } \\
0.65(\mathrm{~mm}) \\
\text { Feed per tooth }(\mathrm{mm})\end{array}$}} \\
\hline & & & & \\
\hline & 0.05 & 0.075 & 0.05 & 0.075 \\
\hline \multirow{3}{*}{$\begin{array}{c}7,000 \\
\ldots \ldots \\
59,500\end{array}$} & $\bullet$ & $\bullet$ & $\bullet$ & $\bullet$ \\
\hline & $\bullet$ & $\bullet$ & $\bullet$ & $\bullet$ \\
\hline & $\bullet$ & $\bullet$ & $\bullet$ & $\bullet$ \\
\hline \multicolumn{5}{|c|}{ LX1(rpm): $7-9-10-13-15-17-19-21-23-25-27-30-35-40-43-45-50-55-59.5$ (66 tests } \\
\hline \multicolumn{5}{|c|}{ FX5(rpm): 7-9-11-13-15-17-19-21-23-25-27 (48 tests) } \\
\hline \multicolumn{5}{|c|}{$\begin{array}{l}\text { Makino(rpm): 4-5-7-8-9-10-11-12-13-14-14.5-15 (43 tests) } \\
(\mathrm{rpm} \times 1000)\end{array}$} \\
\hline
\end{tabular}


The model was built using two principal components with the following percentage explanation: $R^{2} X$ (cum): $0.50, R^{2} Y$ (cum): $0.50, Q^{2}$ (cum): 0.443 . Again the model was built using only normal data (sharp tool) from the three machines. Figure 8 shows the score plot of the results from the null space model inversion and the optimization solutions at $\mathrm{Ra}=0.3 \mu \mathrm{m}$.

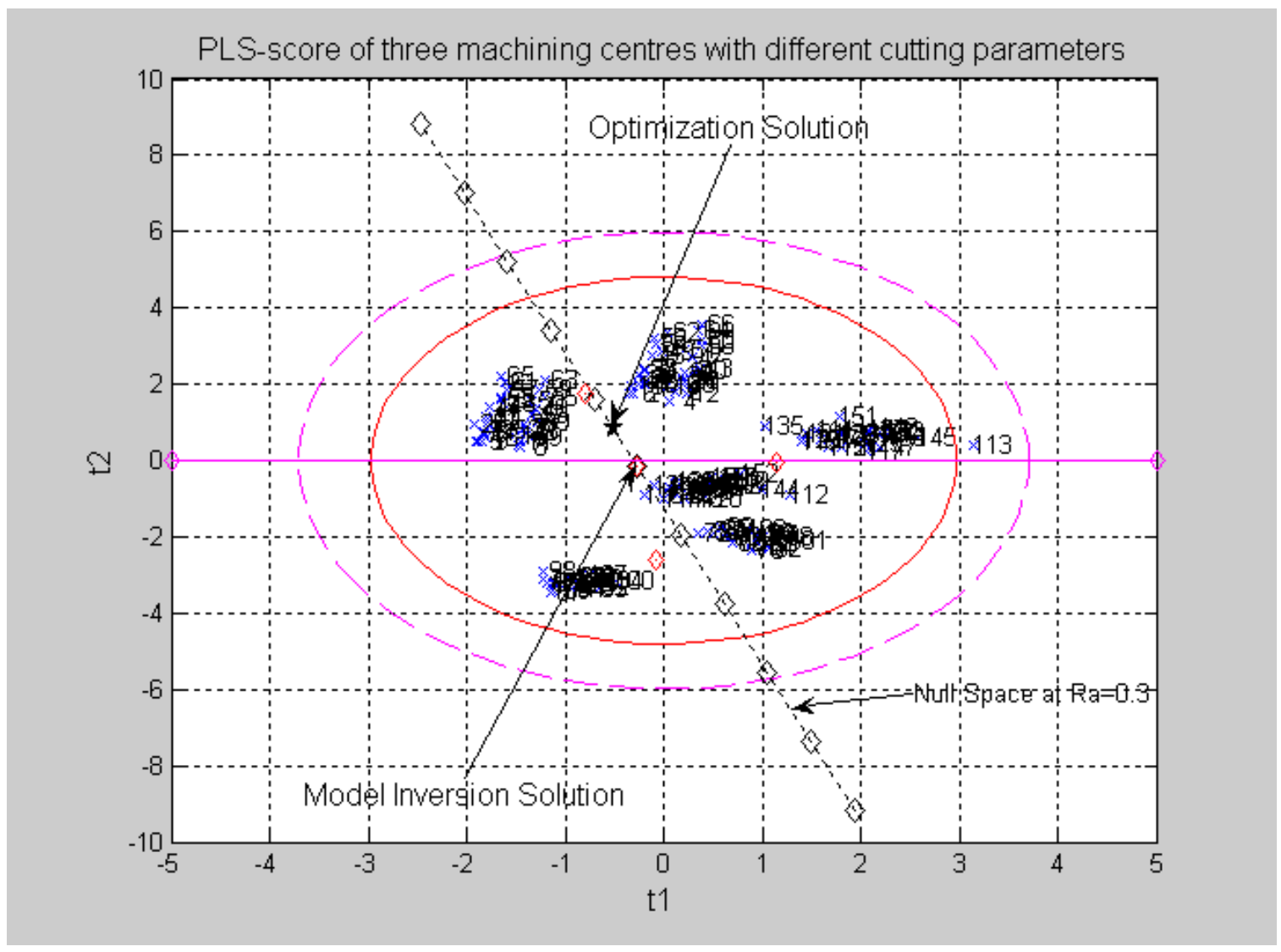

Figure 8. Score plot of data from three machines with optimization and model inversion solutions for $\mathrm{Ra}=0.3 \mu \mathrm{m}$

This optimization solution is almost equivalent to the model inversion solution. Although the model predictability decreased after the addition of different speeds from the machine tools but it still can be used to facilitate the choice of cutting parameters between the different machines. The null space vectors can be used to facilitate the choice of the main cutting parameter for this specific $\mathrm{Ra}=0.3 \mu \mathrm{m}$ by calculating the different possible solutions using the null space vectors as shown in table 7 . The results shown correspond to null space vectors near each group of machine observations. Table 8 shows the cutting parameters from the different training observations from each group of machine observations near the calculated null space for an $\mathrm{Ra}=0.3 \mu \mathrm{m}$.

These observations can facilitate the direct choice, from a group of suitable machines, to carry out the job and in the same time facilitate the choice of cutting parameters to achieve the desired surface roughness. 
Table 7. Model Inversion solutions

\begin{tabular}{|l|c|c|c|}
\hline \multicolumn{4}{|c|}{ Model inversion null space vectors for $\mathrm{Ra}=0.3 \mu \mathrm{m}$} \\
\hline & Feed/tooth $(\mathrm{mm})$ & Depth Of Cut $(\mathrm{mm})$ & $\mathrm{rpm}$ \\
\hline V5 (Makino) & 0.0593 & 0.5513 & 10.8034 \\
\hline V6 (FX5) & 0.0600 & 0.5455 & 19.1531 \\
\hline V7 (LX1) & 0.0606 & 0.5396 & 27.5029 \\
\hline
\end{tabular}

Table 8. Observations from each machine with associated $\mathrm{Ra}$.

$\begin{array}{ccccc}\text { Observation \# } & \begin{array}{c}\text { Feed/tooth } \\ (\mathrm{mm})\end{array} & \begin{array}{r}\text { Depth Of Cut } \\ (\mathrm{mm})\end{array} & \text { rpm } & \text { Ra }(\mu \mathrm{m}) \\ \text { LX1 (59) } & 0.05 & 0.65 & 50,000 & 0.243 \\ \text { LX1 (63) } & 0.05 & 0.65 & 55,000 & 0.269 \\ \text { FX5 (116) } & 0.05 & 0.65 & 7,000 & 0.294 \\ \text { FX5 (134) } & 0.05 & 0.45 & 17,000 & 0.284 \\ \text { Mac (72) } & 0.075 & 0.45 & 7,000 & 0.3 \\ \text { Mac (88) } & 0.075 & 0.45 & 11,000 & 0.301\end{array}$

\section{Model Inversion Solution Using Null Space Vector With Maximum MRR}

In this section, a new idea to facilitate the choice of cutting parameters for a specific Ra is introduced which maximizes the metal removal rate (MRR). MRR is calculated from the following equation:

$$
M R R=\frac{f / t \cdot A D O C \cdot R D O C \cdot r p m \cdot z}{1000}\left(\mathrm{~mm}^{3} / \mathrm{min}\right)
$$

where $z$ is the number of teeth, $f / t$ is the feed per tooth $(\mathrm{mm})$, ADOC is the axial depth of cut $(\mathrm{mm}), \mathrm{RDOC}$ is the radial depth of cut $(\mathrm{mm})$ and $\mathrm{rpm}$ is the rotational speed. The idea is the null space gives a set of solutions (cutting parameters) for the same surface roughness $\mathrm{Ra}$. Then, by calculating MRR as the product of only three variables (cutting parameters) from the PLS surface roughness model, one can get an index for the MRR. In other words, the PLS model consists of 12 variables (sensor outputs, three dummy variables and cutting parameters namely feed/tooth, speed and radial depth of cut) related to the output variable surface roughness $\mathrm{Ra}$. By multiplying feed/tooth, speed and radial depth of cut, an index for the metal removal rate can be found. Then, by constraining the null space solutions inside the Hotelling's limits of $95 \%$ or $99 \%$, one can calculate the MRR for these solutions taking the product of the three cutting parameters. The final step is to extract the maximum value of MRR for all feasible solutions (null space solution inside the Hotelling's T2 limit). Projecting the solution with the maximum MRR index back into the latent space one can find the model inversion solution for a specific $\mathrm{Ra}$ and with the maximum MRR index. This is described as follows:

- The operating parameters $\hat{x}_{n e w}^{T}$ are computed after the estimation of the model scores. 


$$
y_{\text {des }}^{T}=u_{\text {new }}^{T} \cdot \sum_{A} \cdot B \Rightarrow \hat{u}_{\text {new }}^{T}=y_{\text {des }}^{T} \cdot B^{T} \cdot\left(B \cdot B^{T}\right)^{-1} \cdot \sum_{A}^{-1} \Rightarrow \hat{x}_{\text {new }}^{T}=\hat{u}_{\text {new }}^{T} \cdot \sum_{A} \cdot P_{A}^{T}
$$

Then the null space $u_{\text {null }}$ is computed from: $u_{\text {null }}^{T}=\lambda^{T} \cdot G_{2}^{T}$, where $\lambda^{T}$ is arbitrary in magnitude and direction, and solutions are calculated for different values of the $\lambda^{T}$ vectors:

$$
x_{\text {pred }}^{T}=\hat{x}_{\text {new }}^{T}+x_{\text {null }}^{T} \Rightarrow x_{\text {pred }}^{T}=\left(\hat{u}_{\text {new }}^{T}+u_{\text {null }}^{T}\right) \cdot \Sigma_{A} \cdot P_{A}^{T}
$$

Only solutions that are consistent with the past database are retained by checking the Hotelling's confidence limits and set the other equal to zero:

- First calculate the t-scores of the null space solutions: $\quad t_{\text {null }}=x_{\text {pred }}^{T} \cdot w^{*}$

- Computing the Hotelling's $T^{2}$ for each solutions and check for validity:

$$
T^{2}=\sum_{a=1}^{A} \frac{t_{n u l l, a}^{2}}{S_{a}^{2}} \leq T^{2} \lim 95
$$

A Metal Removal Rate index $(M R R i)$ is calculated for all the retained solutions:

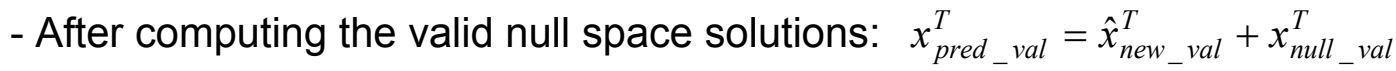

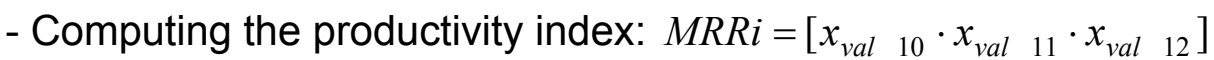

where: " $x_{v a l_{1} 10}, x_{v a l_{11}}, x_{v a l_{1} 12}$ " represents the feed/tooth, depth of cut and the speed in the solution vectors. By taking the maximum value of these indices: $\mathrm{MRRi}_{\max }=\max \left[x_{v a l_{-} 10} \cdot x_{v_{a l} 11} \cdot x_{v a l_{-} 12}\right] ;$ a unique solution is computed and projected back in the latent space to support machine tool selection decisions: $t_{\text {max }_{-} M R R}=x_{\max _{-} M R R} \cdot w^{*}$.

Figure 9 illustrates the idea using the null space solutions at $\mathrm{Ra}=0.3 \mu \mathrm{m}$. It shows the direct model inversion solution and then the model inversion solution with maximum MRR index for the same surface roughness $\mathrm{Ra}=0.3 \mu \mathrm{m}$. Figure 10 shows the loading plot for this model and illustrates how the cutting parameters feed/tooth (Ft), speed (RPM) and depth of cut (DOC) are correlated with each other and with the surface roughness $\mathrm{Ra}$.

Figure 11 shows another solution for the "null space" at $\mathrm{Ra}=0.25 \mu \mathrm{m}$. It is clear the movement of the maximum MRR solution depends on the desired $\mathrm{Ra}$ and the maximum MRR index (upper right quarter of the score plot). The point here is that no feasible solution (inside T2 limit) lies in the lower right quarter which represents the highest MRR index for this null space. On the other hand, the lower left quarter represents the lowest MRR index; this comes from a comparison with the loading plot which shows the distribution of all variables with each other in which can be superimposed at the same time with the score plot. This means that any variable positioned in a specific quarter in the loading plot affects the observations in the same quarter in the score plot. The place where the variable is shown in the loading plot represents the higher value of this variable. 


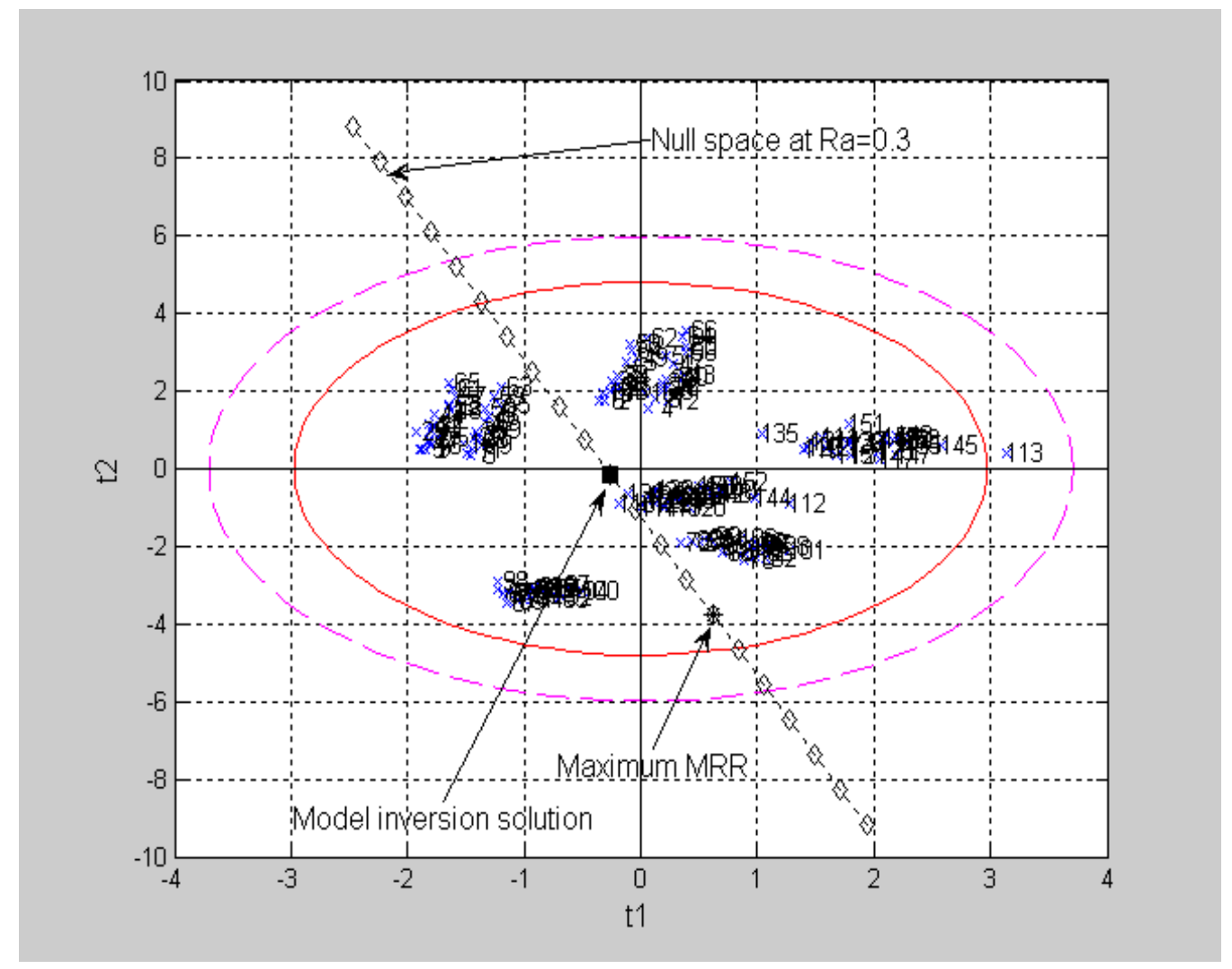

Figure 9. Score plot of data from three machines with model inversion solution and maximum MRR for $\mathrm{Ra}=0.3 \mu \mathrm{m}$

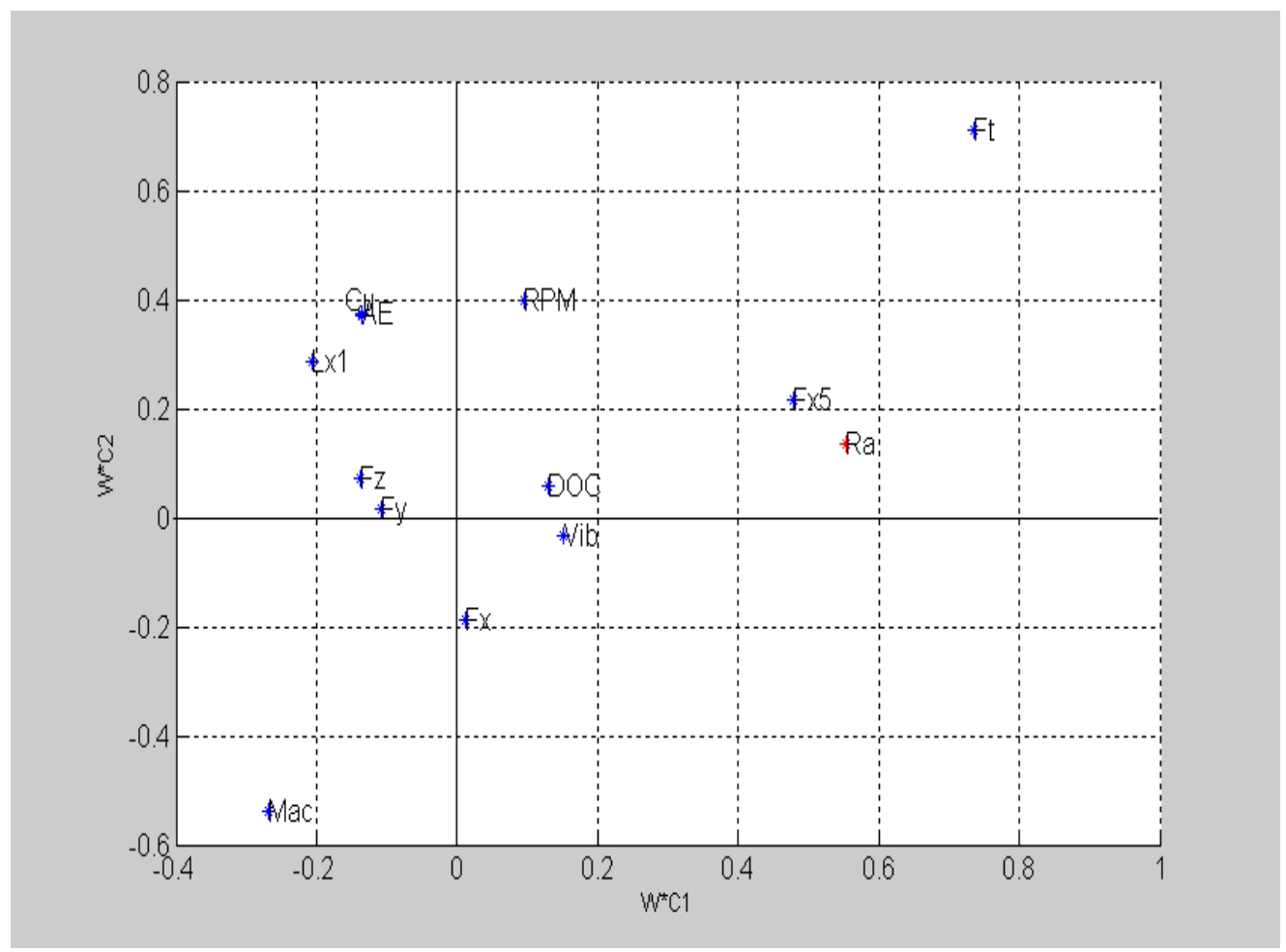

Figure 10. Loading plot of data from three machines. 


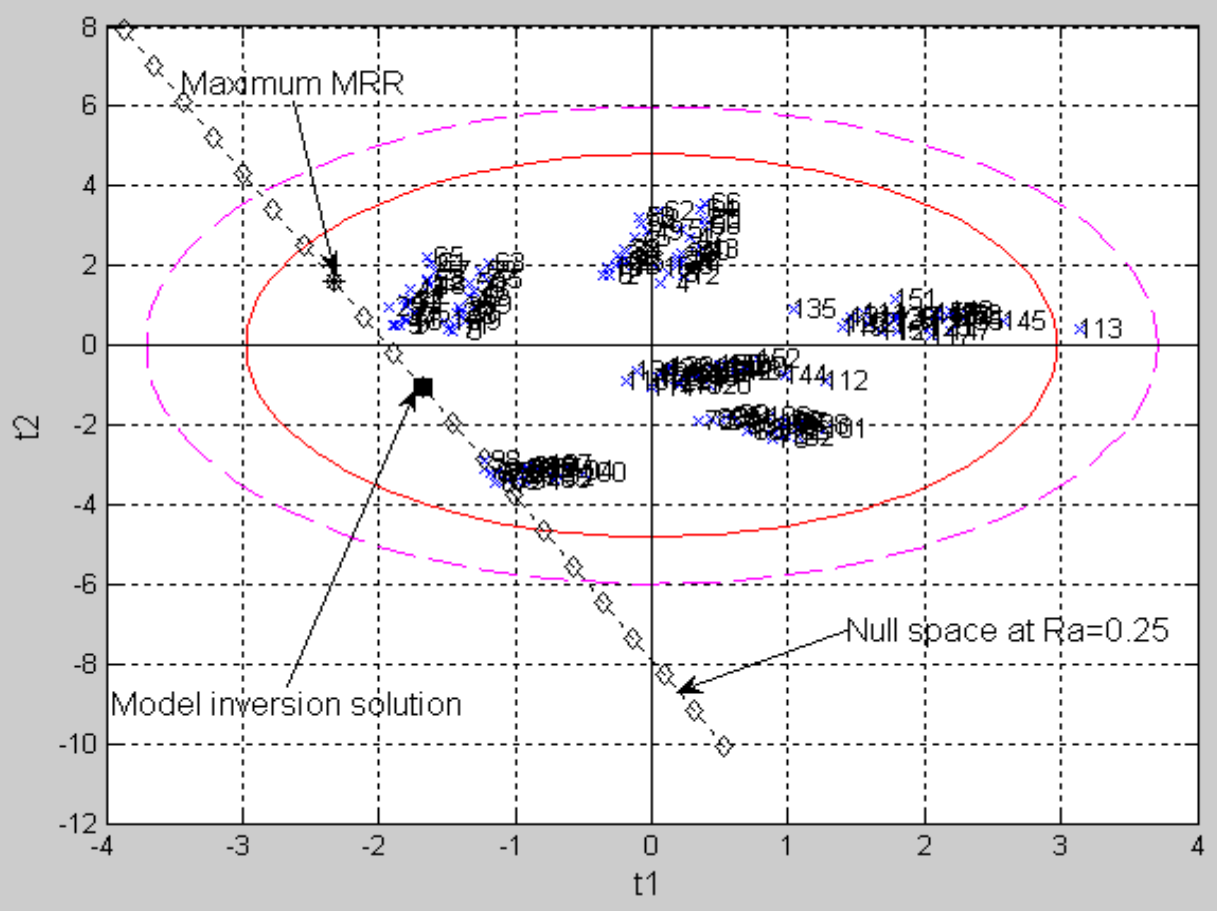

Figure 11. Score plot of data from three machines with model inversion solution and maximum MRR for $\mathrm{Ra}=0.25 \mu \mathrm{m}$

\section{CONCLUSIONS}

Currently there are large amounts of historical data related to manufacturing available in factories for many products. This is due to advances in computer technology and improvements in the robustness of sensors. In this chapter the design approach proposed by Jaeckle and MacGregor $[23,24]$ was applied to machining data associated with an end milling process. Data from a single machining process as well as from three machining centers were used for analysis to arrive at a new set of process conditions to achieve a desired surface roughness $\mathrm{Ra}$. The model inversion approach was formulated as an optimization problem to get a unique solution with constraints. This application facilitates process planning in estimating cutting parameters for desired quality requirements along with the capability to run the process on different machine tools. Also, a new idea was introduced to maximize the production rate over the estimated cutting parameters. In this case a metal removal rate index was calculated for all the feasible null space solutions, inside the T2 limit, using the product of the cutting parameters of these solutions. Then the maximum value for the metal removal rate was extracted and assigned as the process parameter solution. By this approach, the desired $\mathrm{Ra}$ is achieved with the highest MRR. This solution can be projected back in the latent space to facilitate the choice over the existing machining centers. The results show the merit of the approach for this application in the field of machining process planning using the proposed sensory system along with the main cutting parameters. 


\section{REFERENCES}

1. Abdou, G., Tereshkovich, W., "Optimal Operating Parameters in High-Speed Milling Operations for Aluminum," Int. J. Prod. Res., Vol. 39, No. 10, 2001, pp. 2197-2214.

2. Kopac J., Bahor M., Sokovic M., "Optimal machining parameters for achieving the desired surface roughness in fine turning cold pre-formed steel work processing", Int. Mach Tool Manu, 2002, 42:707-716.

3. Sundaram R.M., Lambert B.K., "Mathematical models to predict surface finish in fine turning of steel", Int J Prod Res, 1981, 19:557-564, Part 2.

4. El-Baradie M.A., "Surface roughness model for turning grey cast iron(154 HN)", 1993,Pro. Inst. Mech. Eng., 207.

5. Y. Sahin, A. Motorcu, Surface roughness model for machining mild steel with coated carbide tool, 2005, J. Materials and Design 26 321-326.

6. A. Mansour, H. Abdalla, Surface roughness model for end milling: a semi-free cutting carbon casehardening steel(EN32) in dry condition, 2002, J. Materials Processing Technology 124 183-191.

7. J. Chen, L. Samson, An On-line surface roughness recognition system using an accelerometer in turning operations, 2003, Journal of Engineering Technology.

8. M. Lou, J. Chen, Surface roughness prediction technique for CNC endmilling, 1999, J. Industrial Technology, Vol.15 - 1.

9. D. Baek, T. Ko, H. Kim, A dynamic surface roughness model for face milling, 1997, J. Precision Engineering, 20:171-178.

10. L. Huang, J.Chen, A multiple regression model to predict in-process surface roughness in turning operation via accelerometer, 2001, J. Industrial Technology, Vol.17 - 2 .

11. Suresh, P.V.S., Rao, P.V., Deshmukh, S.G., "A Genetic Algorithm approach for Optimization of Surface Roughness Prediction Model," Int. J. Machine Tools and Manufacture, Vol. 42, 2002, p. 675-680.

12. Fuh, K.-H., Chang, H. Y., "An accuracy model for the peripheral milling of aluminum alloys using response design", J. Mater. Pro. Tech., 1997, 72:42-47.

13. Elbestawi, M.A., Ismail, F., Yuen, K.M., "Surface Topography Characterization in Finish Milling," Int. J. Mach. Tools Manu., Vol. 34, No. 2, 1994, p. 245-255.

14. Hashmi K., El-Baradie M.A., Ryan M., " Development of a fuzzy logic based expert system softwae for intelligent selection of machining parameters", Proceedings of the $22^{\text {nd }}$ international conference on computers and industrial engineering, 1997.

15. Turkay D., Huseyin F.I., Ihsan S.A., "An integrated approach for simultaneous determination of optimum cutting parameters and tools in process planning", Proceedings of the $22^{\text {nd }}$ international conference on computers and industrial engineering, 1997.

16. Azouzi, R., and Guillot, M., 1997, "On-Line Prediction of Surface Finish and Dimensional Deviation in Turning Using Neural Network Based Sensor Fusion,"Int. J. Mach. Tools Manuf., 37, pp. 1201-1217.

17. Tsai, Y. H., Chen, J. C., and Lou, S. J., 1999, "An In-Process Surface Recognition System Based on Neural Networks in End Milling Cutting Operation," Int. J. Mach. Tools Manuf., 39, pp. 583-605.

18. Martellotti, M.E., "An Analysis of the Milling Process,c Transactions of the ASME, Vol. 63, 1941, p 667-700. 
19. Shaw, M.C., Metal Cutting Principles, Oxford, 1997.

20. Montgomery, D., Altintas, Y., "Mechanism of Cutting Force and Surface Generation in Dynamic Milling," Trans. ASME, Vol. 113, 1991, p. 160-168.

21. Lee K., Ahn S.H., Dornfeld D., "A study of surface roughness in the microend-milling process", Consortium on deburring and egde finishing Univ. of California, Berkeley, 2004.

22. Moteki Y., Arai Y., "Operation planning and quality design of a polymer process", DYCORD IFAC Symposium, Bournemouth-UK, December 1986.

23. C. Jaeckle and J. MacGregor, "Product design through multivariate statistical analysis of process data", AICHE, May 1998

24. C. Jaeckle and J. MacGregor, "Industrial applications of product design through the inversion of latent variable models", Chemometrics and intelligent laboratory systems, 2000, 199-210

25. F. Yacoub and J. MacGregor, "Product optimization and control in the latent variable space of nonlinear PLS models", Chemometrics and intelligent laboratory systems, 2004, 63-74

26. S.G. Munoz, J. MacGregor and T. Kourti, "Product transfer between sites using joint-Y PLS ", Chemometrics and intelligent laboratory systems, 2005, 101114

27. Hussein W.M., Veldhuis S.C., Elbestawi M.A., "Tool wear monitoring for next generation e-factory Using Multivariate Statistical Models", Proceeding of the CIRP $2^{\text {nd }}$ International conference on high performance cutting, 2006, Vancouver. 